\title{
The Garnir relations for Weyl groups of type $C_{n}$
}

\author{
Himmet Can \\ Department of Mathematics, Faculty of Arts \& Sciences \\ Erciyes University, 38039 Kayseri, Turkey \\ can@erciyes.edu.tr
}

Submitted: Nov 10, 2007; Accepted: May 16, 2008; Published: May 26, 2008

Mathematics Subject Classifications: 20F55, 20C30

\begin{abstract}
The Garnir relations play a very important role in giving combinatorial constructions of representations of the symmetric groups. For the Weyl groups of type $C_{n}$, having obtained the alternacy relation, we give an explicit combinatorial description of the Garnir relation associated with a $\Delta$-tableau in terms of root systems. We then use these relations to find a $K$-basis for the Specht modules of the Weyl groups of type $C_{n}$.
\end{abstract}

\section{Introduction}

Although a great deal of progress has been made in generalizing the representation theory of symmetric groups to Weyl groups, very little has been done using the combinatorial approach. The first attempt at providing such a generalization has been given by Morris [14], where the basic combinatorial concepts such as tableau, tabloid, etc., which were successful for symmetric groups as exemplified in the work of James [13], were interpreted in the context of root systems of Weyl groups. In recent years, a further development of these ideas has appeared in Halicioglu and Morris [10] and Halicioglu [8]. In this alternative approach, the Weyl groups of type $A_{n}$ and $C_{n}$ are used to motivate a possible generalization to Weyl groups in general.

For the construction of a basis for the Specht modules of Weyl groups, Halicioglu [8] has considered the root systems of simply laced type only (i.e., $A_{n}, D_{n}, E_{6}, E_{7}, E_{8}$ ) and their parabolic subsystems. Later, the present author [4] extended these ideas to deal with the root systems of type $C_{n}$. Having obtained the 'perfect systems', Halicioglu [8] and the present author [4] conclude that the set of standard $\Delta$ - polytabloids is a basis. But they do not prove that standard $\Delta$ - polytabloids span the Specht module $S^{\Delta, \Delta^{\prime}}$. Inspired by the work of Peel [15], Halicioglu [9] introduced the Garnir relations for Weyl groups. But he does not prove that standard $\Delta$ - polytabloids span $S^{\Delta, \Delta^{\prime}}$. That is, no counterparts of Theorems 1.1, 3.1 and 3.4 in [15] are given in his work. 
The main object of this paper is to construct the Garnir relations in terms of the root systems of type $C_{n}$ in a form which may be taken as a role model for the root systems of other Weyl groups. Indeed, at the end of this paper, by using the proposed method here we illustrate how a Garnir relation can be constructed for the root systems of type $D_{n}$. We hope to extend these ideas to the Weyl group of any type in the future. The structure of the paper will be as follows. In the first section we develop the needed notation and give the necessary basic facts about the Specht modules $S^{\Delta, \Delta^{\prime}}$. We introduce the very good systems in Section 2 to obtain a linearly independent subset of the $S^{\Delta, \Delta^{\prime}}$. Here, our approach follows closely that due to Halicioglu [8]. In the final section, we construct the Garnir relations for the Weyl groups of type $C_{n}$ so that the standard $\Delta$-polytabloids span $S^{\Delta, \Delta^{\prime}}$.

\section{Preliminaries}

We first establish the basic notation and state some results which are required later. We refer the reader to [10] and [4] for much of the undefined terminology and quoted results.

1.1 Let $\Phi$ be a root system relating to the Weyl group $W=W(\Phi)$ with simple system $\pi$ and corresponding positive system $\Phi^{+}$. Let $\Psi$ be a subsystem of $\Phi$ with simple system $J \subset \Phi^{+}$and Dynkin diagram $\Delta$. If $\Psi=\sum_{i=1}^{k} \Psi_{i}$, where $\Psi_{i}$ are the indecomposable components of $\Psi$, then let $J_{i}$ be a simple system in $\Psi_{i}(i=1, \ldots, k)$ and $J=\sum_{i=1}^{k} J_{i}$. Let $\Psi^{\perp}$ be the largest subsystem in $\Phi$ orthogonal to $\Psi$ and let $J^{\perp} \subset \Phi^{+}$be the simple system of $\Psi^{\perp}$. Let $\Psi^{\prime}$ be a subsystem of $\Phi$ which is contained in $\Phi \backslash \Psi$, with simple system $J^{\prime} \subset \Phi^{+}$ and Dynkin diagram $\Delta^{\prime}$. If $\Psi^{\prime}=\sum_{i=1}^{l} \Psi_{i}^{\prime}$, where $\Psi_{i}^{\prime}$ are the indecomposable components of $\Psi^{\prime}$, then let $J_{i}^{\prime}$ be a simple system in $\Psi_{i}^{\prime}(i=1, \ldots, l)$ and $J^{\prime}=\sum_{i=1}^{l} J_{i}^{\prime}$. Let $\Psi^{\prime \perp}$ be the largest subsystem in $\Phi$ orthogonal to $\Psi^{\prime}$ and let $J^{\prime \perp} \subset \Phi^{+}$be the simple system of $\Psi^{\prime \perp}$. Let $\bar{J}$ stand for the ordered set $\left\{J_{1}, \ldots, J_{k} ; J_{1}^{\prime}, \ldots, J_{l}^{\prime}\right\}$, where in addition the elements in each $J_{i}$ and $J_{i}^{\prime}$ are ordered, and put $\mathcal{T}_{\Delta}=\{w \bar{J} \mid w \in W\}$. The pair $\bar{J}=\left\{J, J^{\prime}\right\}$ is called a useful system in $\Phi$ if $W(J) \cap W\left(J^{\prime}\right)=\langle e\rangle$ and $W\left(J^{\perp}\right) \cap W\left(J^{\perp}\right)=\langle e\rangle$. Let $\bar{J}_{1}$ and $\bar{J}_{2}$ be useful systems in $\Phi$. We say that $\bar{J}_{1}$ is $W$-conjugate to $\bar{J}_{2}$ if there exists $w \in W$ such that $\bar{J}_{2}=w \bar{J}_{1}$. The elements of $\mathcal{T}_{\Delta}$ are called $\Delta$-tableaux, the $J_{i}$ and $J_{i}^{\prime}$ are called the rows and columns of the useful system respectively. This construction is a natural extension of the concept of a Young tableau in the representation theory of symmetric groups (for a fuller explanation, see [10]). We may also interpret this for the special case $W\left(C_{n}\right)$ with the help of the work of [14] as follows. 
1.2 Let $\Phi=C_{n}$ with simple system $\pi=\left\{\alpha_{i}=e_{i}-e_{i+1}(i=1, \ldots, n-1), \alpha_{n}=\right.$ $\left.2 e_{n}\right\}$. By [7], let $\Psi=\sum_{i=1}^{r} A_{\lambda_{i}}+\sum_{j=1}^{s} C_{\mu_{j}}\left(\sum_{i=1}^{r}\left(\lambda_{i}+1\right)+\sum_{j=1}^{s} \mu_{j}=n\right)$, then let $J_{\lambda_{i}}^{(1)}$ and $J_{\mu_{j}}^{(2)}$ be simple systems in $A_{\lambda_{i}}(i=1, \ldots, r)$ and $C_{\mu_{j}}(j=1, \ldots, s)$ respectively and $J=J^{(1)}+J^{(2)}$, where $J^{(1)}=\sum_{i=1}^{r} J_{\lambda_{i}}^{(1)}$ and $J^{(2)}=\sum_{j=1}^{s} J_{\mu_{j}}^{(2)}$. Let $\Psi^{\prime}=\sum_{i=1}^{r^{\prime}} C_{\lambda_{i}^{\prime}}+$ $\sum_{j=1}^{s^{\prime}} A_{\mu_{j}^{\prime}}\left(\sum_{i=1}^{r^{\prime}} \lambda_{i}^{\prime}+\sum_{j=1}^{s^{\prime}}\left(\mu_{j}^{\prime}+1\right)=n\right)$, then let $J_{\lambda_{i}^{\prime}}^{(1)}$ and $J_{\mu_{j}^{\prime}}^{(2)}$ be simple systems in $C_{\lambda_{i}^{\prime}}(i=$ $\left.1, \ldots, r^{\prime}\right)$ and $A_{\mu_{j}^{\prime}}\left(j=1, \ldots, s^{\prime}\right)$ respectively and $J^{\prime}=J^{\prime(1)}+J^{\prime(2)}$, where $J^{\prime(1)}=$ $\sum_{i=1}^{r^{\prime}} J_{\lambda_{i}^{\prime}}^{\prime(1)}$ and $J^{\prime(2)}=\sum_{j=1}^{s^{\prime}} J_{\mu_{j}^{\prime}}^{\prime(2)}$. Inspired by the concept of a double Young tableau in [14], we identify $\bar{J}$ with the ordered double set $\left\{\left(J^{(1)} ; J^{\prime(1)}\right),\left(J^{(2)} ; J^{\prime(2)}\right)\right\}$ given by

$$
\left\{\left(J_{\lambda_{1}}^{(1)}, \ldots, J_{\lambda_{r}}^{(1)} ; J_{\lambda_{1}^{\prime}}^{\prime(1)}, \ldots, J_{\lambda_{r^{\prime}}^{\prime}}^{\prime(1)}\right),\left(J_{\mu_{1}}^{(2)}, \ldots, J_{\mu_{s}}^{(2)} ; J_{\mu_{1}^{\prime}}^{(2)}, \ldots, J_{\mu_{s^{\prime}}^{\prime}}^{(2)}\right)\right\}
$$

where in addition the elements in each $J_{\lambda_{i}}^{(1)}, J_{\mu_{j}}^{(2)}, J_{\lambda_{i}^{\prime}}^{\prime(1)}$ and $J_{\mu_{j}^{\prime}}^{(2)}$ are ordered. Namely, for $\lambda_{1} \geq \lambda_{2} \geq \cdots \geq \lambda_{r} \geq 0, \mu_{1} \geq \mu_{2} \geq \cdots \geq \mu_{s} \geq 1$ and $\sum_{i=1}^{r}\left(\lambda_{i}+1\right)+\sum_{j=1}^{s} \mu_{j}=$ $n$, let $\Psi=\sum_{i=1}^{r} A_{\lambda_{i}}+\sum_{j=1}^{s} C_{\mu_{j}}$ be a subsystem of $\Phi$ then $(\lambda, \mu)=\left(\lambda_{1}+1, \ldots, \lambda_{r}+\right.$ $\left.1, \mu_{1}, \ldots, \mu_{s}\right)$ is a pair of partitions of $n$, and so the corresponding Weyl subgroup is $W\left(A_{\lambda_{1}}\right) \times \cdots \times W\left(A_{\lambda_{r}}\right) \times W\left(C_{\mu_{1}}\right) \times \cdots \times W\left(C_{\mu_{s}}\right)$ which is isomorphic to the subgroup $\mathcal{S}_{\lambda_{1}+1} \times \cdots \times \mathcal{S}_{\lambda_{r}+1} \times O_{\mu_{1}} \times \cdots \times O_{\mu_{s}}$ of the hyperoctahedral group $O_{n}$. Put $k_{0}=0, k_{i}=$ $\lambda_{1}+\cdots+\lambda_{i}+i(i=1, \ldots, r)$ and $l_{0}=k_{r}=\sum_{i=1}^{r}\left(\lambda_{i}+1\right), l_{j}=l_{0}+\mu_{1}+\cdots \mu_{j}(j=1, \ldots, s)$, then

$$
\begin{aligned}
J_{k_{i}}^{(1)} & =\left\{\alpha_{k_{i-1}+1}, \alpha_{k_{i-1}+2}, \ldots, \alpha_{k_{i}-1}\right\} \\
& =\left\{e_{k_{i-1}+1}-e_{k_{i-1}+2}, e_{k_{i-1}+2}-e_{k_{i-1}+3}, \ldots, e_{k_{i}-1}-e_{k_{i}}\right\}
\end{aligned}
$$

is a simple system for $A_{\lambda_{i}}$ and therefore $J^{(1)}=\sum_{i=1}^{r} J_{k_{i}}^{(1)}$ is a simple system for $\sum_{i=1}^{r} A_{\lambda_{i}}$, and

$$
\begin{aligned}
J_{l_{j}}^{(2)} & =\left\{\alpha_{l_{j-1}+1}, \alpha_{l_{j-1}+2}, \ldots, \alpha_{l_{j}-1}, 2 e_{l_{j}}\right\} \\
& =\left\{e_{l_{j-1}+1}-e_{l_{j-1}+2}, e_{l_{j-1}+2}-e_{l_{j-1}+3}, \ldots, e_{l_{j}-1}-e_{l_{j}}, 2 e_{l_{j}}\right\}
\end{aligned}
$$


is a simple system for $C_{\mu_{j}}$ and therefore $J^{(2)}=\sum_{j=1}^{s} J_{l_{j}}^{(2)}$ is a simple system for $\sum_{j=1}^{s} C_{\mu_{j}}$. Thus, $J=J^{(1)}+J^{(2)}$ is a simple system for $\Psi=\sum_{i=1}^{r} A_{\lambda_{i}}+\sum_{j=1}^{s} C_{\mu_{j}}$, and the subsystem $\Psi$ may be represented by the rows of the $(\lambda, \mu)$-tableau

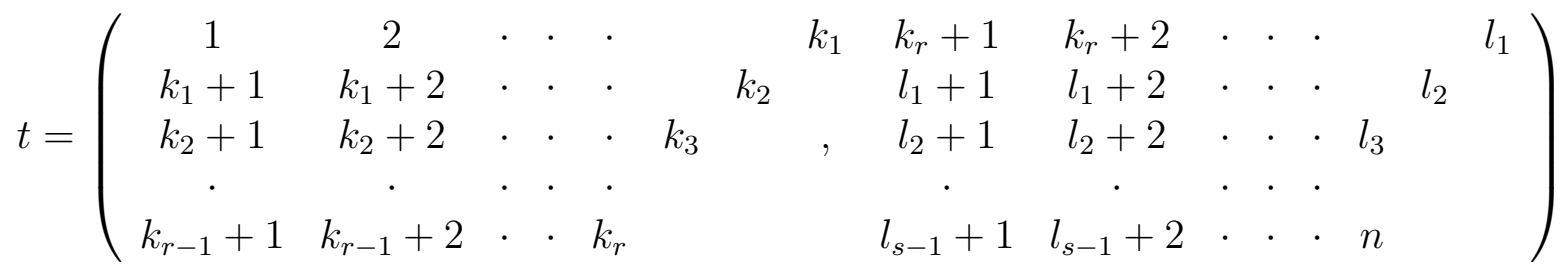

as in [14], the other $2^{n} n !(\lambda, \mu)$-tableaux being obtained by allowing the elements of $O_{n}$ to act on this tableau. The orthogonal subsystem $\Psi^{\perp}$ is the root system determined by the elements in rows of length one in the first part of the $(\lambda, \mu)$-tableau $t$. Let $\Psi^{\prime}=$ $\sum_{i=1}^{r^{\prime}} C_{\lambda_{i}^{\prime}}+\sum_{j=1}^{s^{\prime}} A_{\mu_{j}^{\prime}}$ be the subsystem of $\Phi$ with simple system $J^{\prime}=J^{\prime(1)}+J^{\prime(2)}$, where $J^{\prime}=J^{\prime(1)}+J^{\prime(2)}$ is represented by the columns of the $(\lambda, \mu)$-tableau $t$ (in [4], we showed how to determine the $J^{\prime}$ ). Then the orthogonal subsystem $\Psi^{\prime \perp}$ is the root system determined by the elements in columns of length one in the second part of the $(\lambda, \mu)$-tableau $t$. Hence, $W(J) \cong R_{t}$ and $W\left(J^{\prime}\right) \cong C_{t}$, where $R_{t}$ (resp. $\left.C_{t}\right)$ is the row (resp. column) stabilizer of the $(\lambda, \mu)$-tableau $t$. Since $W(J) \cap W\left(J^{\prime}\right)=\langle e\rangle$ and $W\left(J^{\perp}\right) \cap W\left(J^{\perp}\right)=\langle e\rangle$ then $\bar{J}=\left\{\left(J^{(1)} ; J^{\prime(1)}\right),\left(J^{(2)} ; J^{\prime(2)}\right)\right\}$ is a useful system in $\Phi$. The $J_{\lambda_{i}}^{(1)}$ and $J_{\lambda_{i}^{\prime}}^{\prime(1)}\left(J_{\mu_{j}}^{(2)}\right.$ and $\left.J_{\mu_{j}^{\prime}}^{(2)}\right)$ are called the rows and columns of the first part (second part) of the useful system respectively. Note that there are, of course, useful systems that are not $W$-conjugate to any of the useful systems corresponding to bipartitions.

1.3 Two $\Delta$-tableaux $\bar{J}$ and $\bar{K}$ are row equivalent, written $\bar{J} \sim \bar{K}$, if there exists $w \in W(J)$ such that $\bar{K}=w \bar{J}$. The equivalence class which contains the $\Delta$-tableaux $\bar{J}$ is $\{\bar{J}\}$ and is called a $\Delta$-tabloid. Let $\tau_{\Delta}$ be the set of all $\Delta$-tabloids, then we have $\tau_{\Delta}=$ $\left\{\{w \bar{J}\} \mid w \in D_{\Psi}\right\}$, where $D_{\Psi}=\left\{w \in W \mid w(\alpha) \in \Phi^{+}\right.$for all $\left.\alpha \in J\right\}$ is a distinguished set of coset representatives for $W(\Psi)$ in $W$ (see [12]). The Weyl group $W$ acts on $\tau_{\Delta}$ according to $\sigma\{w \bar{J}\}=\{\sigma w \bar{J}\}$ for all $\sigma \in W$. Let $K$ be an arbitrary field and let $M^{\Delta}$ be the $K$-space whose basis elements are the $\Delta$-tabloids. Extending this action to be linear on $M^{\Delta}$ turns $M^{\Delta}$ into a $K W$-module. Define $\kappa_{\bar{J}} \in K W$ and $e_{\bar{J}}$ by $\kappa_{\bar{J}}=\sum_{\sigma \in W\left(J^{\prime}\right)}(\operatorname{sgn} \sigma) \sigma$ and $e_{\bar{J}}=$ $\kappa_{\bar{J}}\{\bar{J}\}$, where sgn $\sigma=(-1)^{l(\sigma)}$ with $l(\sigma)$ being the length of $\sigma$. Then $e_{\bar{J}}$ is called the $\Delta$ polytabloid associated with $\bar{J}$. The Specht module $S^{\Delta, \Delta^{\prime}}$ is the submodule of $M^{\Delta}$ generated by $e_{w \bar{J}}$, where $w \in W$. A useful system $\bar{J}$ in $\Phi$ is called a good system if $w \Psi \cap \Psi^{\prime}=\emptyset$ for $w \in D_{\Psi}$ then $\{w \bar{J}\}$ appears in $e_{\bar{J}}$. If $\bar{J}$ is a good system in $\Phi$ and the characteristic of $K$ is zero, then $S^{\Delta, \Delta^{\prime}}$ is irreducible.

As in the case of the symmetric group, generally the $\Delta$-polytabloids that generate $S^{\Delta, \Delta^{\prime}}$ are not linearly independent. Therefore, it would be nice to determine a subset 
which forms a basis for $S^{\Delta, \Delta^{\prime}}$-e.g., for computing the matrices and characters of the representation.

In the next section, we shall consider how the definition of a good system can be modified so that the set $B^{\Delta, \Delta^{\prime}}=\left\{e_{w \bar{J}} \mid w \bar{J}\right.$ is a standard $\Delta$-tableau $\}$ is linearly independent over $K$.

\section{Linear independence}

In the symmetric groups, in order to determine a $K$-basis for the Specht modules, standard tableaux, tabloids and polytabloids are defined. We now define the counterparts in the more general context of root systems and Weyl groups. In this section, our approach will follow closely that due to Halicioglu [8].

Let $\bar{J}$ be a good system in $\Phi$, and $w \in W$. A $\Delta$ - tableau $w \bar{J}$ is row standard (resp.column standard) if $w \in D_{\Psi}$ (resp. $w \in D_{\Psi^{\prime}}$ ). A $\Delta$-tableau $w \bar{J}$ is standard if $w \in D_{\Psi} \cap D_{\Psi^{\prime}}$. A $\Delta$-tabloid $\{w \bar{J}\}$ is standard if there is a standard $\Delta$-tableau in the equivalence class $\{w \bar{J}\}$. A $\Delta$-polytabloid $e_{w \bar{J}}$ is standard if $w \bar{J}$ is standard. Thus, if $w \bar{J}$ is row standard (resp. column standard), then $w J \subset \Phi^{+}\left(\right.$resp. $\left.w J^{\prime} \subset \Phi^{+}\right)$. Also, if $w \bar{J}$ is standard, then $w J \subset \Phi^{+}$and $w J^{\prime} \subset \Phi^{+}$.

To establish that the set $B^{\Delta, \Delta^{\prime}}$ is linearly independent over $K$, we shall need a partial order on $\Delta$-tabloids. Following Humphreys [11], the Bruhat order on the elements of a Weyl group is defined as follows. Let $w, w^{\prime} \in W$ and $\alpha \in \Phi^{+}$. Write $w \stackrel{\alpha}{\rightarrow} w^{\prime}$ if $w^{\prime}=s_{\alpha} w$ and $l(w)<l\left(w^{\prime}\right)$, where $l(w)$ denotes the length of $w$. Then define $w<w^{\prime}$ if there exists a chain $w=w_{0} \stackrel{\alpha_{1}}{\rightarrow} w_{1} \stackrel{\alpha_{2}}{\rightarrow} \cdots \stackrel{\alpha_{m}}{\rightarrow} w_{m}=w^{\prime}$, where $\alpha_{1}, \ldots, \alpha_{m} \in \Phi^{+}$. It is clear that the resulting relation $w \leq w^{\prime}$ is a partial ordering of $W$, with $e$ as the unique minimal element. We call it the Bruhat ordering. Thus we have that $w<w^{\prime}$ if there exist $\alpha_{1}, \ldots, \alpha_{m} \in \Phi^{+}$ such that $w^{\prime}=s_{\alpha_{m}} \ldots s_{\alpha_{1}} w$ and $l\left(s_{\alpha_{i-1}} \ldots s_{\alpha_{1}} w\right)<l\left(s_{\alpha_{i}} \ldots s_{\alpha_{1}} w\right)$ for all $i=1, \ldots, m$. We now use this partial order on $W$ in order to define a partial order on $\Delta$-tabloids. It is clear that the Bruhat order $\leq$ on $W$ will also be a partial order when restricted to $D_{\Psi}$.

Now, let $\bar{J}$ be a good system in $\Phi$ and let $w, w^{\prime} \in D_{\Psi}$. Then $\left\{w^{\prime} \bar{J}\right\}$ dominates $\{w \bar{J}\}$, written $\{w \bar{J}\} \unlhd\left\{w^{\prime} \bar{J}\right\}$ if and only if $w \leq w^{\prime}$. Clearly $\unlhd$ is a partial order on $\Delta$-tabloids.

A good system $\bar{J}$ is called a very good system in $\Phi$ if $w \leq w^{\prime}$ for all $w \in D_{\Psi} \cap D_{\Psi^{\prime}}$, $w^{\prime} \in D_{\Psi}$ such that $w^{\prime}=w \sigma \rho$, where $\sigma \in W\left(J^{\prime}\right), \rho \in W(J)$. With this definition, we have the following.

Lemma 2.1 Let $\bar{J}$ be a very good system in $\Phi$ and let $w, w^{\prime} \in D_{\Psi}$. If $w \bar{J}$ is a standard tableau and $\left\{w^{\prime} \bar{J}\right\}$ appears in $e_{w \bar{J}}$ then $\{w \bar{J}\} \unlhd\left\{w^{\prime} \bar{J}\right\}$.

Proof See Lemma 3.7 [8].

The previous lemma says that $\{w \bar{J}\}$ is the minimum tabloid in $e_{w \bar{J}}$.

Lemma 2.2 Let $v_{1}, v_{2}, \ldots, v_{m}$ be elements of $M^{\Delta}$. Suppose, for each $v_{i}$, we can choose a tabloid $\left\{w_{i} \bar{J}\right\}$ appearing in $v_{i}$ such that

(i) $\left\{w_{i} \bar{J}\right\}$ is the minimum in $v_{i}$, and 
(ii) the $\left\{w_{i} \bar{J}\right\}$ are all distinct.

Then $\left\{v_{1}, v_{2}, \ldots, v_{m}\right\}$ is linearly independent over $K$.

Proof See Lemma 3.8 [8].

Lemma 2.2 corresponds to Lemma 2.5.8 in Sagan [16].

Proposition 2.3 If $\bar{J}$ is a very good system in $\Phi$, then the set $B^{\Delta, \Delta^{\prime}}=\left\{e_{w \bar{J}} \mid w \bar{J}\right.$ is a standard $\Delta-$ tableau\} is linearly independent over $K$.

Proof By Lemma 2.1, $\{w \bar{J}\}$ is minimum in $e_{w \bar{J}}$, and by hypothesis they are all distinct. Thus Lemma 2.2 can be applied to complete the proof.

Thus, for a Weyl group, if we have a very good system $\bar{J}$ in $\Phi$ then the set $B^{\Delta, \Delta^{\prime}}$ is linearly independent over $K$. But the question arises whether this set is a $K$-basis for $S^{\Delta, \Delta^{\prime}}$. In that case, a very good system $\bar{J}$ is called a perfect system in $\Phi$ if the set $B^{\Delta, \Delta^{\prime}}$ is a $K$ basis for $S^{\Delta, \Delta^{\prime}}$.

Example 2.4 Let $\Phi=C_{3}$ with simple system $\pi=\left\{\alpha_{i}=e_{i}-e_{i+1}(i=1,2), \alpha_{3}=2 e_{3}\right\}$. Let $w_{\alpha_{i}}$ be denoted by $w_{i}, i=1,2,3$. Let $\Psi=C_{2}+C_{1}$ be a subsystem of $C_{3}$ with simple system $J=\left\{e_{1}-e_{2}, 2 e_{2}, 2 e_{3}\right\}$. Then $W(J)=\left\langle w_{1}, w_{2} w_{3} w_{2}\right\rangle \times\left\langle w_{3}\right\rangle$ and $D_{\Psi}=$ $\left\{e, w_{2}, w_{1} w_{2}\right\}$. In this case the possible good systems in $\Phi$ are

(i) $\left\{J, J_{1}^{\prime}\right\}$, where $\Psi_{1}^{\prime}=A_{1}$ with simple system $J_{1}^{\prime}=\left\{e_{1}-e_{3}\right\}$,

(ii) $\left\{J, J_{2}^{\prime}\right\}$, where $\Psi_{2}^{\prime}=A_{1}$ with simple system $J_{2}^{\prime}=\left\{e_{1}+e_{3}\right\}$,

(iii) $\left\{J, J_{3}^{\prime}\right\}$, where $\Psi_{3}^{\prime}=A_{1}$ with simple system $J_{3}^{\prime}=\left\{e_{2}-e_{3}\right\}$,

(iv) $\left\{J, J_{4}^{\prime}\right\}$, where $\Psi_{4}^{\prime}=A_{1}$ with simple system $J_{4}^{\prime}=\left\{e_{2}+e_{3}\right\}$.

In case (ii) $D_{\Psi} \cap D_{\Psi_{2}^{\prime}}=D_{\Psi}$ and $W\left(J_{2}^{\prime}\right)=\left\langle w_{1} w_{3} w_{2} w_{3} w_{1}\right\rangle$. Now, let $w=w_{1} w_{2} \in$ $D_{\Psi} \cap D_{\Psi_{2}^{\prime}}$ and $w^{\prime}=w_{2} \in D_{\Psi}$. Then there exist $\sigma=w_{1} w_{3} w_{2} w_{3} w_{1} \in W\left(J_{2}^{\prime}\right)$ and $\rho=$ $w_{1} w_{2} w_{3} w_{2} w_{1} w_{3} \in W(J)$ such that $w^{\prime}=w \sigma \rho$. But $w \not \leq w^{\prime}$. Hence $\left\{J, J_{2}^{\prime}\right\}$ is not a very good system in $\Phi$. Similarly it can be verified that $\left\{J, J_{4}^{\prime}\right\}$ is not a very good system in $\Phi$.

In case (i) $D_{\Psi} \cap D_{\Psi_{1}^{\prime}}=\left\{e, w_{2}\right\}$ and $W\left(J_{1}^{\prime}\right)=\left\langle w_{2} w_{1} w_{2}\right\rangle$. Now let $w=w_{2} \in D_{\Psi} \cap D_{\Psi_{1}^{\prime}}$ and let $w^{\prime}=w_{2} \in D_{\Psi}$. Then there exist $\sigma=e \in W\left(J_{1}^{\prime}\right)$ and $\rho=e \in W(J)$ such that $w^{\prime}=w \sigma \rho$. Then $w=w^{\prime}$. Let $w=w_{2} \in D_{\Psi} \cap D_{\Psi_{1}^{\prime}}$ and $w^{\prime}=w_{1} w_{2} \in D_{\Psi}$. Then there exist $\sigma=w_{2} w_{1} w_{2} \in W\left(J_{1}^{\prime}\right)$ and $\rho=e \in W(J)$ such that $w^{\prime}=w \sigma \rho$. Then $w<w^{\prime}$. Hence, $\left\{J, J_{1}^{\prime}\right\}$ is a very good system in $\Phi$. Similarly it can be verified that $\left\{J, J_{3}^{\prime}\right\}$ is also a very good system in $\Phi$ ( since $\left.D_{\Psi} \cap D_{\Psi_{3}^{\prime}}=\{e\}\right)$.

The very good system $\left\{J, J_{1}^{\prime}\right\}$ corresponds to the one constructed in (1.2) for the bipartition $(\lambda, \mu)=(\emptyset, 21)$, and so we have the isomorphism $S^{J, J_{1}^{\prime}} \cong S^{\lambda, \mu}$. Also, by Proposition 3.9 of [10], we have $S^{J, J_{3}^{\prime}} \cong S^{J, J_{1}^{\prime}}$. But $\left\{J, J_{3}^{\prime}\right\}$ is not a perfect system, since there is only one standard tableau corresponding to $D_{\Psi} \cap D_{\Psi_{3}^{\prime}}=\{e\}$ whereas $S^{J, J_{3}^{\prime}} \cong S^{\lambda, \mu}$ has dimension 2, where $(\lambda, \mu)=(\emptyset, 21)$. In the next section, we show that $\left\{J, J_{1}^{\prime}\right\}$ is a perfect system in $\Phi$. 
As seen in Example 2.4, note that not all the useful systems (resp. good systems, very good systems) are good system (resp. very good system, perfect system).

For the special case $W\left(C_{n}\right)$, the useful systems constructed in (1.2) can be translated to the language of $(\lambda, \mu)$-tableaux in the hyperoctahedral groups context; that is, the key concepts (i.e., the useful systems, good systems, very good systems and perfect systems) are reduced to the standard $(\lambda, \mu)$-tableaux for the systems constructed in (1.2). Thus, in these cases, there are isomorphisms between the Specht modules $S^{\Delta, \Delta^{\prime}}$ and the Specht modules $S^{\lambda, \mu}$ given in [1], which send the $\Delta$-polytabloids (resp. standard polytabloids) to the $(\lambda, \mu)$-polytabloids (resp. standard polytabloids). Therefore, if char $K=0$ then the $S^{\Delta, \Delta^{\prime}}$ give a complete set of irreducible $K W$-modules (cf. Theorem 2.6 of [1] or Theorem 3.21 of [2]). In the following section, we shall give the Garnir relations for the systems constructed in (1.2) only.

\section{Garnir relations for type $C_{n}$}

Let $\Phi$ be a root system associated with $W=W\left(C_{n}\right)$. We now show that standard $\Delta$ polytabloids span $S^{\Delta, \Delta^{\prime}}$; that is, if $w \bar{J}$ is an arbitrary $\Delta$-tableau, where $w \in W$, then $e_{w \bar{J}}$ is a linear combination of standard $\Delta$-polytabloids.

To determine the Garnir element of $w \bar{J}$ associated with $e_{w \bar{J}}$, we use the following relations which correspond to the work in [1].

Lemma 3.1 Let $\bar{J}$ be a very good system in $\Phi$. Let $w \bar{J}$ be a $\Delta$-tableau, where $w \in W$. If $\alpha$ is any root in $w J^{\prime}$, then

$$
\left.\left(e+w_{\alpha}\right) e_{w \bar{J}}=0 \quad \text { (alternacy relation }\right)
$$

Proof Let $\alpha \in w J^{\prime}$. Then $\alpha \in \Phi$, and so $\alpha=w_{\alpha_{1}} \ldots w_{\alpha_{k}}(\beta)$ for suitable roots $\alpha_{1}, \ldots, \alpha_{k}, \beta \in \pi$, by 2.1 .8 of [5]. Thus $w_{\alpha}=w_{\alpha_{1}} \ldots w_{\alpha_{k}} w_{\beta} w_{\alpha_{k}} \ldots w_{\alpha_{1}}$, and so sgn $w_{\alpha}=-1$. Since $w_{\alpha} \in W\left(w J^{\prime}\right)$, the result follows immediately from $w_{\alpha} e_{w \bar{J}}=$ $\left(\operatorname{sgn} w_{\alpha}\right) e_{w \bar{J}}=-e_{w \bar{J}}$.

Note that we have used no special properties of $\Phi$ in the proof of Lemma 3.1, so the result remains true for any root system.

Remark 3.2 By Lemma 3.10 of [10], if $w=d \rho$, where $d \in D_{\Psi^{\prime}}$ and $\rho \in W\left(J^{\prime}\right)$, then we have $e_{w \bar{J}}=(\operatorname{sgn} \rho) e_{d \bar{J}}$. Hence one can always assume that $w \in D_{\Psi^{\prime}}$, which means that $w \bar{J}$ is column standard.

Now, let $\bar{J}$ be a very good system in $\Phi$ with notation as in (1.2). Let $w \bar{J}$ be a $\Delta$-tableau, where $w \in W$. Suppose that $w \bar{J}$ is column standard but not row standard. Then $\beta \in \Phi^{-}$ for some $\beta \in w J$. If $\beta=-2 e_{i}$ for some $i$, then $\beta \in w J^{(2)}$. Let $\pi \in W\left(w J^{\prime}\right)$. Then $w_{\beta} \pi=\pi w_{\pi^{-1}(\beta)}$ and $\pi^{-1}(\beta)$ appears in $W\left(w J^{(2)}\right) w J^{(2)}$, so that $w_{\pi^{-1}(\beta)} \in W(w J)$ and 


$$
\begin{aligned}
w_{\pi^{-1}(\beta)}\{w \bar{J}\}=\{w \bar{J}\} . \text { Thus, } & \\
w_{\beta} e_{w \bar{J}} & =\sum_{\pi \in W\left(w J^{\prime}\right)}(\operatorname{sgn} \pi) w_{\beta} \pi\{w \bar{J}\} \\
& =\sum_{\pi \in W\left(w J^{\prime}\right)}(\operatorname{sgn} \pi) \pi w_{\pi^{-1}(\beta)}\{w \bar{J}\}=e_{w \bar{J}}
\end{aligned}
$$

Therefore, we have the following lemma.

Lemma 3.3 Let $\bar{J}$ be a very good system in $\Phi$ with notation as in (1.2) and $w \bar{J}$ be a $\Delta$-tableau, where $w \in W$. Suppose that $w \bar{J}$ is column standard but not row standard. If $\beta=-2 e_{i}$ appears in $w J^{(2)}$ for some $i$, then

$$
\left.\left(e-w_{\beta}\right) e_{w \bar{J}}=0 \quad \text { (sign change relation }\right) .
$$

Remark 3.4 The previous two lemmas say that we can find the elements of $W$ which make $w \bar{J}$ column standard (alternacy relation) and which turn any negative long roots $-2 e_{i}$ of $w J$ associated with $e_{w \bar{J}}$ into positive long roots (sign change relation), i.e., the tableau $w \bar{J}$ associated with $e_{w \bar{J}}$ may be reorganized so that all columns are standard and no negative long roots remain in $w J$. Note that at this point, alternacy relations, unlike sign change relations, are direct consequences of the definition of the polytabloids.

Example 3.5 Let $\Phi=C_{7}$ with simple system $\pi=\left\{\alpha_{i}=e_{i}-e_{i+1}(i=1,2, \ldots, 6), \alpha_{7}=\right.$ $\left.2 e_{7}\right\}$ and corresponding Weyl group $W=W(\Phi)$. Let $w_{\alpha_{i}}$ be denoted by $w_{i}, i=$ $1,2, \ldots, 7$. Let $\Psi=A_{1}+A_{1}+C_{2}+C_{1}$ be a subsystem of $C_{7}$ with simple system $J=J^{(1)}+J^{(2)}=\left\{e_{1}-e_{2}, e_{3}-e_{4}\right\} \cup\left\{e_{5}-e_{6}, 2 e_{6}, 2 e_{7}\right\}$. Then the corresponding Dynkin diagram $\Delta$ for $\Psi$ is

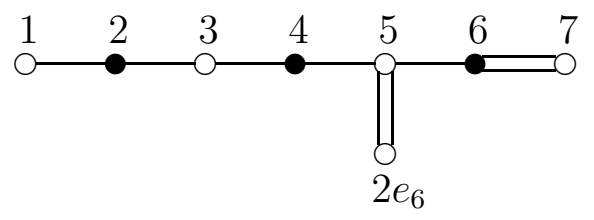

where the nodes corresponding to $\alpha_{1}, \ldots, \alpha_{7}$ are denoted by $1, \ldots, 7$ respectively, the nodes $2,4,6$ have been deleted and the node $2 e_{6}$ has been added. On the other hand, the subsystem $\Psi=A_{1}+A_{1}+C_{2}+C_{1}$ corresponds to the pair of partitions $(\lambda, \mu)=(22,21)$ of 7. Thus the subsystem $\Psi=A_{1}+A_{1}+C_{2}+C_{1}$ is represented by the rows of the tableau

$$
t=\left(\begin{array}{llll}
1 & 2 & 5 & 6 \\
3 & 4 & 7 &
\end{array}\right)
$$

as in [14]. Now by applying Algorithm 3.1 of [4], the subsystem of $\Phi$ which is contained in $\Phi \backslash \Psi$ is obtained to be $\Psi^{\prime}=C_{2}+C_{2}+A_{1}$ with simple system $J^{\prime}=J^{\prime(1)}+J^{\prime(2)}=$ $\left\{e_{1}-e_{3}, 2 e_{3}, e_{2}-e_{4}, 2 e_{4}\right\} \cup\left\{e_{5}-e_{7}\right\}$. This means that Algorithm 3.1 of [4] enables us to construct the subsystem $\Psi^{\prime}$ such that its simple system $J^{\prime}$ is represented by the columns of the above tableau $t$. Thus, it follows from the discussion in Section 2 that

$$
\bar{J}=\left\{\left(e_{1}-e_{2}, e_{3}-e_{4} ; e_{1}-e_{3}, 2 e_{3}, e_{2}-e_{4}, 2 e_{4}\right),\left(e_{5}-e_{6}, 2 e_{6}, 2 e_{7} ; e_{5}-e_{7}\right)\right\}
$$


is a very good system in $\Phi$. If $w=w_{2} w_{3} w_{7} \in W$, then

$$
w \bar{J}=\left\{\left(e_{1}-e_{3}, e_{4}-e_{2} ; e_{1}-e_{4}, 2 e_{4}, e_{3}-e_{2}, 2 e_{2}\right),\left(e_{5}-e_{6}, 2 e_{6},-2 e_{7} ; e_{5}+e_{7}\right)\right\}
$$

is a $\Delta$-tableau. Since the root $\alpha=e_{3}-e_{2}$ is in $w J^{\prime}$ and the root $\beta=-2 e_{7}$ appears in $w_{3} w_{7} J^{(2)}$, then we have

$$
\begin{aligned}
e_{w \bar{J}}=-w_{\alpha} e_{w \bar{J}} & =-e_{w_{3} w_{7} \bar{J}} \text { (alternacy relation) } \\
& =-w_{\beta} e_{w_{3} w_{7} \bar{J}} \\
& =-e_{w_{3} \bar{J}} \text { (sign change relation) }
\end{aligned}
$$

Now we shall find elements of the group algebra of $W$ which annihilate the given $\Delta$ polytabloid $e_{w \bar{J}}$. Let $w \in W$, and let $w \bar{J}$ be a $\Delta$-tableau associated with $e_{w \bar{J}}$ such that the entries of $w \bar{J}$ were reorganized by the alternacy relations so that all columns were standard. Suppose that $w \bar{J}$ is not row standard. Then there must be some negative roots in $w J$. For example, for the root $\alpha^{*} \in w J$, say $\alpha^{*} \in \Phi^{-}$. Then we know that $-\alpha^{*} \in \Phi^{+}$. Now, define $J^{-\alpha^{*}}=\left\{\gamma \in w J^{\prime} \mid\left(\gamma,-\alpha^{*}\right) \leq 0\right\}$ and $J_{-\alpha^{*}}=\left\{-\alpha^{*}\right\} \cup J^{-\alpha^{*}}$. Then we have the following proposition.

Proposition 3.6 The set $J_{-\alpha^{*}}$ is linearly independent over $\mathbf{R}$. Furthermore, $J_{-\alpha^{*}}$ yields a subsystem of $\Phi$.

Proof Let $J_{-\alpha^{*}}=\left\{\gamma_{1}, \ldots, \gamma_{k}\right\}$ with $\gamma_{1}=-\alpha^{*}$ and $J^{-\alpha^{*}}=\left\{\gamma_{2}, \ldots, \gamma_{k}\right\}$. Then by definition of the set $J_{-\alpha^{*}}$, we have $\left(\gamma_{i}, \gamma_{j}\right) \leq 0$ for all $i \neq j$. Suppose that $J_{-\alpha^{*}}$ is linearly dependent over $\mathbf{R}$, i.e., let $\sum_{i=1}^{k} a_{i} \gamma_{i}=0$ be a non-trivial relation.

Put $M=\left\{i \mid a_{i}>0\right\}$ and $N=\left\{i \mid a_{i}<0\right\}$, and write $\lambda_{i}=a_{i}, i \in M$ and $\mu_{i}=-a_{i}, i \in N$. Then

$$
\gamma=\sum_{i \in M} \lambda_{i} \gamma_{i}=\sum_{j \in N} \mu_{j} \gamma_{j} \neq 0
$$

where $\lambda_{i}, \mu_{j}>0$ for all $i \in M$ and $j \in N$. But we have

$$
0<(\gamma, \gamma)=\sum_{i, j} \lambda_{i} \mu_{j}\left(\gamma_{i}, \gamma_{j}\right) \leq 0
$$

This forces $\gamma=0$ which is a contradiction. Thus $J_{-\alpha^{*}}$ must be linearly independent over R.

Now, denote by $W\left(J_{-\alpha^{*}}\right)$ the group generated by all reflections $w_{\gamma_{i}}$ with $\gamma_{i} \in J_{-\alpha^{*}}$, $i=1, \ldots, k$, then $W\left(J_{-\alpha^{*}}\right)$ is a subgroup of $W$ and so $W\left(J_{-\alpha^{*}}\right)$ is a finite reflection group. Thus, by (4.2) of [6] $J_{-\alpha^{*}}$ is a root graph. Let $\Psi_{-\alpha^{*}}=W\left(J_{-\alpha^{*}}\right) J_{-\alpha^{*}}$, then the set $\Psi_{-\alpha^{*}}$ is the pre-root system corresponding to $J_{-\alpha^{*}}$ with $W\left(\Psi_{-\alpha^{*}}\right)=W\left(J_{-\alpha^{*}}\right)$ by $(4.10)$ (i) of [6]. But, by (4.11) (ii) of [6] the set $\Psi_{-\alpha^{*}}$ is a root system and so is a subsystem of $\Phi$. Hence, we have the required result. 
By (1.4) of [3], we say that $\Psi_{-\alpha^{*}}$ is a subsystem of $\Phi$ with simple system $J_{-\alpha^{*}} \subset \Phi^{+}$. We know that $W\left(J_{-\alpha^{*}}\right)$ and $W\left(w J^{\prime}\right)$ are subgroups of $W$. Now, define $S=W\left(J_{-\alpha^{*}}\right) \cap W\left(w J^{\prime}\right)$, and so $S$ is a subgroup of $W\left(J_{-\alpha^{*}}\right)$. Let $\sigma_{1}, \ldots, \sigma_{r}$ be coset representatives for $S$ in $W\left(J_{-\alpha^{*}}\right)$, and let

$$
W\left(J_{-\alpha^{*}}\right)=\biguplus_{j=1}^{r} \sigma_{j} S \text { and } \mathcal{G}_{w \bar{J}}=\sum_{j=1}^{r}\left(\operatorname{sgn} \sigma_{j}\right) \sigma_{j} .
$$

$\mathcal{G}_{w \bar{J}}$ is called a Garnir element associated with $w \bar{J}$.

Remark 3.7 The coset representatives $\sigma_{1}, \ldots, \sigma_{r}$ are, of course, not unique, but for practical purposes note that we may take $\sigma_{1}, \ldots, \sigma_{r}$ so that $\sigma_{1} w \bar{J}, \ldots, \sigma_{r} w \bar{J}$ are all the column standard tableaux.

Example 3.8 Referring to Example 3.5, we have $e_{w \bar{J}}=-e_{w_{3}} \bar{J}$. Since $\alpha^{*}=e_{4}-e_{3}$ is a negative root in $w_{3} J$,

$$
w_{3} \bar{J}=\left\{\left(e_{1}-e_{2}, e_{4}-e_{3} ; e_{1}-e_{4}, 2 e_{4}, e_{2}-e_{3}, 2 e_{3}\right),\left(e_{5}-e_{6}, 2 e_{6}, 2 e_{7} ; e_{5}-e_{7}\right)\right\}
$$

is not row standard. Now, put $J^{-\alpha^{*}}=\left\{\gamma \in w_{3} J^{\prime} \mid\left(\gamma,-\alpha^{*}\right) \leq 0\right\}=\left\{2 e_{4}, e_{2}-e_{3}, e_{5}-e_{7}\right\}$ and $J_{-\alpha^{*}}=\left\{-\alpha^{*}\right\} \cup J^{-\alpha^{*}}=\left\{e_{2}-e_{3}, e_{3}-e_{4}, 2 e_{4}, e_{5}-e_{7}\right\}$. By Proposition 3.6, $\Psi_{-\alpha^{*}}=C_{3}+A_{1}$ is a subsystem of $\Phi$ with simple system $J_{-\alpha^{*}}$ and Dynkin diagram

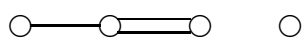

In this case, $W\left(J_{-\alpha^{*}}\right)=\left\langle w_{2}, w_{3}, w_{4} w_{5} w_{6} w_{7} w_{6} w_{5} w_{4}\right\rangle \times\left\langle w_{5} w_{6} w_{5}\right\rangle, W\left(w_{3} J^{\prime}\right)=$ $\left\langle w_{1} w_{2} w_{3} w_{2} w_{1}, \quad w_{4} w_{5} w_{6} w_{7} w_{6} w_{5} w_{4}\right\rangle \times\left\langle w_{2}, \quad w_{3} w_{4} w_{5} w_{6} w_{7} w_{6} w_{5} w_{4} w_{3}\right\rangle \times\left\langle w_{5} w_{6} w_{5}\right\rangle$ and $S=W\left(J_{-\alpha^{*}}\right) \cap W\left(w_{3} J^{\prime}\right)=\left\langle w_{2} w_{3} w_{4} w_{5} w_{6} w_{7} w_{6} w_{5} w_{4} w_{3} w_{2}\right\rangle \times\left\langle w_{3} w_{4} w_{5} w_{6} w_{7} w_{6} w_{5} w_{4} w_{3}\right\rangle \times$ $\left\langle w_{4} w_{5} w_{6} w_{7} w_{6} w_{5} w_{4}\right\rangle \times\left\langle w_{5} w_{6} w_{5}\right\rangle \times\left\langle w_{2}\right\rangle$.

Let $e, w_{3}, w_{2} w_{3}$ be coset representatives for $S$ in $W\left(J_{-\alpha^{*}}\right)$. Then $\mathcal{G}_{w_{3} \bar{J}}=e-w_{3}+w_{2} w_{3}$ is the Garnir element associated with $w_{3} \bar{J}$.

Let $H$ be any subset of $W$. Define

$$
\bar{H}=\sum_{\sigma \in H}(\operatorname{sgn} \sigma) \sigma
$$

and if $H=\{\sigma\}$ then we write $\bar{\sigma}=(\operatorname{sgn} \sigma) \sigma$ for $\bar{H}$.

Lemma 3.9 Let $\Upsilon$ be a subsystem of $\Phi$ with simple system $\Gamma$.

(i) If $\alpha$ is any root in $\Upsilon$, then we can factor $\overline{W(\Gamma)}=k\left(e-w_{\alpha}\right)$ for some $k \in K W$.

(ii) If $\bar{J}$ is a useful system in $\Phi$ with the root $\alpha \in \Psi$ such that $w_{\alpha} \in W(\Gamma)$, then $\overline{W(\Gamma)}\{\bar{J}\}=0$. 
Proof (i) Consider the subgroup $P=\left\{e, w_{\alpha}\right\}$ of $W(\Gamma)$. Select coset representatives $\sigma_{1}, \ldots, \sigma_{s}$ for $P$ in $W(\Gamma)$ and write $W(\Gamma)=\biguplus_{i=1}^{s} \sigma_{i} P$. But then

$$
\overline{W(\Gamma)}=\left(\sum_{i=1}^{s} \bar{\sigma}_{i}\right)\left(e-w_{\alpha}\right)
$$

as desired.

(ii) Since $\alpha \in \Psi, w_{\alpha} \in W(J)$ and so $w_{\alpha}\{\bar{J}\}=\{\bar{J}\}$. Thus,

$$
\overline{W(\Gamma)}\{\bar{J}\}=k\left(e-w_{\alpha}\right)\{\bar{J}\}=k(\{\bar{J}\}-\{\bar{J}\})=0 .
$$

Proposition 3.10 Assume that $\bar{J}$ is a very good system in $\Phi$ with notation as in (1.2). Suppose that $w \bar{J}$ is column standard but not row standard, where $w \in W$. Let $J_{-\alpha^{*}}$, $S$ be as in the definition of a Garnir element, and let $\Psi_{-\alpha^{*}}$ be the subsystem of $\Phi$ determined by $J_{-\alpha^{*}}$. If $\pi w \Psi \cap \Psi_{-\alpha^{*}} \neq \emptyset$ for all $\pi \in W\left(w J^{\prime}\right)$, then

$$
\mathcal{G}_{w \bar{J}} e_{w \bar{J}}=0 \quad \text { (Garnir relation) } .
$$

Proof Let

$$
\overline{W\left(J_{-\alpha^{*}}\right)}=\sum_{\sigma \in W\left(J_{-\alpha^{*}}\right)}(\operatorname{sgn} \sigma) \sigma \text { and } \bar{S}=\sum_{\sigma \in S}(\operatorname{sgn} \sigma) \sigma .
$$

Consider any $\pi \in W\left(w J^{\prime}\right)$. Then by the hypothesis, there exists a root $\alpha \in \pi w \Psi$ such that $w_{\alpha} \in W\left(J_{-\alpha^{*}}\right)$. Thus, by Lemma $3.9 \overline{W\left(J_{-\alpha^{*}}\right)}\{\pi w \bar{J}\}=0$. Since this is true for every $\pi$ appearing in $\kappa_{w J^{\prime}}$, we have $\overline{W\left(J_{-\alpha^{*}}\right)} e_{w \bar{J}}=0$.

Now $W\left(J_{-\alpha^{*}}\right)=\biguplus_{j=1}^{r} \sigma_{j} S$, so $\overline{W\left(J_{-\alpha^{*}}\right)}=\mathcal{G}_{w \bar{J}} \bar{S}$. Since $S \subset W\left(w J^{\prime}\right)$ then $\bar{S}$ is a factor of $\kappa_{w J^{\prime}}$ and $\bar{S} e_{w \bar{J}}=|S| e_{w \bar{J}}$. Therefore,

$$
0=\overline{W\left(J_{-\alpha^{*}}\right)} e_{w \bar{J}}=|S| \mathcal{G}_{w \bar{J}} e_{w \bar{J}} .
$$

Thus, $\mathcal{G}_{w \bar{J}} e_{w \bar{J}}=0$ when the base field is $\mathbf{Q}$, and since all the tabloid coefficients here are integers, the same holds over any field $K$.

Remark 3.11 For the negative long roots $-2 e_{i}$, we now show that the Garnir relations are equivalent to the sign change relations. Let $\bar{J}$ be a very good system in $\Phi$ with notation as in (1.2). Suppose that $w \bar{J}$ is column standard but not row standard, where $w \in W$. If we do not use the sign change relation, then an element of $w J \cap \Psi^{-}$can be of the form $-2 e_{i}$ for some $i$, and so $-2 e_{i} \in w J^{(2)}$. Now, put $-\alpha^{*}=2 e_{i}$. Then by definition of the set $J^{-\alpha^{*}}$, all the elements of $w J^{\prime}$ occur in $J^{-\alpha^{*}}$ except for the element $e_{k}+e_{i}$ for some $k$ when $e_{k}+e_{i}$ occurs in $w J^{\prime(2)}$ (for if whenever $e_{k}+e_{i}$ occurs in $w J^{\prime(2)}$ then $\left.\left(e_{k}+e_{i},-\alpha^{*}\right)>0\right)$. Namely, if $e_{k}+e_{i}$ occurs in $w J^{\prime(2)}$ then we have $J^{-\alpha^{*}}=w J^{\prime} \backslash\left\{e_{k}+e_{i}\right\}$ and $J_{-\alpha^{*}}=\left\{-\alpha^{*}\right\} \cup\left(w J^{\prime} \backslash\left\{e_{k}+e_{i}\right\}\right)$. But if $e_{k}+e_{i}$ does not occur in $w J^{\prime(2)}$ then $J^{-\alpha^{*}}=w J^{\prime}$ 
and $J_{-\alpha^{*}}=\left\{-\alpha^{*}\right\} \cup w J^{\prime}$. Thus by Proposition 3.6, the corresponding subsystem for $-\alpha^{*}$ is $\Psi_{-\alpha^{*}}$ with simple system $J_{-\alpha^{*}}$.

Now, consider the subgroup $S=W\left(J_{-\alpha^{*}}\right) \cap W\left(w J^{\prime}\right)$. Then by construction of the $J_{-\alpha^{*}}, S$ is a subgroup of $W\left(J_{-\alpha^{*}}\right)$ of index 2 . But then by considering Remark 3.7, the construction of the $W\left(J_{-\alpha^{*}}\right)$ enables us to choose the elements $e$ and $w_{-\alpha^{*}}$ for $S$ in $W\left(J_{-\alpha^{*}}\right)$ as the coset representatives. Hence, $\mathcal{G}_{w \bar{J}}=e-w_{-\alpha^{*}}$ is the Garnir element associated with $w \bar{J}$. Furthermore, by construction of the part $w J^{(2)}$, suppose that we have the long roots $2 e_{i_{1}}, 2 e_{i_{2}}, \ldots, 2 e_{i_{r}}$ in $w \Psi$ ( of course, one of them is $-\alpha^{*}$ since $\left.\alpha^{*} \in w J^{(2)}\right)$. If $\pi \in W\left(w J^{\prime}\right)$, then there exists $2 e_{i_{j}} \in w \Psi$ such that $\pi\left(2 e_{i_{j}}\right)= \pm \alpha^{*}$ for some $j \in\{1,2, \ldots, r\}$. Thus, we always have $\pm \alpha^{*} \in \pi w \Psi \cap \Psi_{-\alpha^{*}}$ for all $\pi \in W\left(w J^{\prime}\right)$, and so by Proposition 3.10 we have the Garnir relation $\mathcal{G}_{w \bar{J}} e_{w \bar{J}}=\left(e-w_{-\alpha^{*}}\right) e_{w \bar{J}}=0$, which turns out to be the sign change relation.

To illustrate this fact, referring to Example 3.5, let $w_{7} \bar{J}=\left\{\left(e_{1}-e_{2}, e_{3}-e_{4} ; e_{1}-e_{3}, 2 e_{3}, e_{2}-e_{4}, 2 e_{4}\right),\left(e_{5}-e_{6}, 2 e_{6},-2 e_{7} ; e_{5}+e_{7}\right)\right\}$

be a $\Delta$-tableau, where $w_{7} \in W$. Then $w_{7} \bar{J}$ is column standard but not row standard. Now, put $-\alpha_{1}^{*}=2 e_{7}$, then we have $J^{-\alpha_{1}^{*}}=\left\{e_{1}-e_{3}, 2 e_{3}, e_{2}-e_{4}, 2 e_{4}\right\}=w_{7} J^{\prime} \backslash\left\{e_{5}+e_{7}\right\}$ and $J_{-\alpha_{1}^{*}}=\left\{-\alpha_{1}^{*}\right\} \cup J^{-\alpha_{1}^{*}}=\left\{e_{1}-e_{3}, 2 e_{3}, e_{2}-e_{4}, 2 e_{4}, 2 e_{7}\right\}$. By Proposition 3.6, $\Psi_{-\alpha_{1}^{*}}=C_{2}+C_{2}+C_{1}$ is a subsystem of $\Phi$ with simple system $J_{-\alpha_{1}^{*}}$. Now take the subgroup $S=W\left(J_{-\alpha_{1}^{*}}\right) \cap W\left(w_{7} J^{\prime}\right)$. By considering Remark 3.7, let $e$ and $w_{-\alpha_{1}^{*}}=w_{7}$ be coset representatives for $S$ in $W\left(J_{-\alpha_{1}^{*}}\right)$. Then the corresponding Garnir element associated with $w_{7} \bar{J}$ is $\mathcal{G}_{w_{7} \bar{J}}=e-w_{7}$. Since $\pm \alpha_{1}^{*} \in \pi w_{7} \Psi \cap \Psi_{-\alpha_{1}^{*}}$ for all $\pi \in W\left(w_{7} J^{\prime}\right)$ then by Proposition 3.10 we have $\left(e-w_{7}\right) e_{w_{7} \bar{J}}=0$, which is the sign change relation. Referring to Example 3.5 once again, let

$w_{6} w_{7} w_{6} \bar{J}=\left\{\left(e_{1}-e_{2}, e_{3}-e_{4} ; e_{1}-e_{3}, 2 e_{3}, e_{2}-e_{4}, 2 e_{4}\right),\left(e_{5}+e_{6},-2 e_{6}, 2 e_{7} ; e_{5}-e_{7}\right)\right\}$

be a $\Delta$-tableau, where $w_{6} w_{7} w_{6} \in W$. Then $w_{6} w_{7} w_{6} \bar{J}$ is column standard but not row standard. Now, take $-\alpha_{2}^{*}=2 e_{6}$, then we have $J^{-\alpha_{2}^{*}}=w_{6} w_{7} w_{6} J^{\prime}$ and $J_{-\alpha_{2}^{*}}=\left\{-\alpha_{2}^{*}\right\} \cup$ $J^{-\alpha_{2}^{*}}=\left\{e_{1}-e_{3}, 2 e_{3}, e_{2}-e_{4}, 2 e_{4}, e_{5}-e_{7}, 2 e_{6}\right\}$. By using a similar argument as above, we have $\mathcal{G}_{w_{6} w_{7} w_{6} \bar{J}}=e-w_{6} w_{7} w_{6}$, where $w_{-\alpha_{2}^{*}}=w_{6} w_{7} w_{6}$, and so $\left(e-w_{6} w_{7} w_{6}\right) e_{w_{6} w_{7} w_{6} \bar{J}}=0$, which means the sign change relation again.

Since the sign change relations are faster in practical calculation, one can use them. But we recall that we shall confine the role of them as a theoretical approach.

Example 3.12 Referring to Example 3.5 and Example 3.8, since $\pi w_{3} \Psi \cap \Psi_{-\alpha^{*}} \neq \emptyset$ for all $\pi \in W\left(w_{3} J^{\prime}\right)$ then we have $0=\mathcal{G}_{w_{3} \bar{J}} e_{w_{3} \bar{J}}=e_{w_{3} \bar{J}}-e_{\bar{J}}+e_{w_{2} \bar{J}}$, so $e_{w_{3} \bar{J}}=e_{\bar{J}}-e_{w_{2} \bar{J}}$, where $w_{2} \in D_{\Psi} \cap D_{\Psi^{\prime}}$. Thus

$$
e_{w \bar{J}}=-e_{w_{3} \bar{J}}=-e_{\bar{J}}+e_{w_{2} \bar{J}}
$$

which if we use the traditional notation as in [14] corresponds to

$$
{ }^{e}\left(\begin{array}{rrrr}
1 & 3 & 5 & 6 \\
4 & 2 & -7 &
\end{array}\right)=-e\left(\begin{array}{llll}
1 & 2 & 5 & 6 \\
3 & 4 & 7 &
\end{array}\right)^{+e}\left(\begin{array}{llll}
1 & 3 & 5 & 6 \\
2 & 4 & 7 &
\end{array}\right) .
$$


Remark 3.13 We now impose a partial order on the column equivalence classes. To define a partial order on the row equivalence classes in Section 2, we have used the $D_{\Psi}$. But note that it is wrong to define the ordering by using $D_{\Psi^{\prime}}$. A partial order on the column equivalence classes may be defined as follows: Let $\bar{J}$ be a very good system in $\Phi$ with notation as in (1.2). Then $\bar{J}$ corresponds to the standard bitableau $t$ given in (1.2). Let $\widetilde{t}$ denote the standard bitableau obtained from the $t$ by interchanging rows and columns, as in a matrix. Now, take another standard $\Delta$-tableau $\widetilde{\bar{J}}$ in $\Phi$ which corresponds to the $\widetilde{t}$ as in (1.2). (This is only for the purpose of defining the ordering on the column equivalence classes; we are still considering the Specht module constructed from the original system $\bar{J}$.) Then $\widetilde{\widetilde{J}}$ is $W$-conjugate to the original system $\bar{J}$. Write $[\widetilde{\bar{J}}]$ for the column equivalence class of $\widetilde{\bar{J}}$; that is, $[\widetilde{\bar{J}}]=\left\{\widetilde{\bar{L}} \mid \widetilde{\bar{L}}=\pi \widetilde{\bar{J}}\right.$ for some $\left.\pi \in W\left(\widetilde{J^{\prime}}\right)\right\}$. Then $\left[w^{\prime} \widetilde{\bar{J}}\right]$ dominates $[w \widetilde{\bar{J}}]$ (where $w, w^{\prime} \in D_{\widetilde{\Psi}^{\prime}}$ ), written $[w \widetilde{\bar{J}}] \unlhd\left[w^{\prime} \widetilde{\bar{J}}\right]$, if $w \leq w^{\prime}$ in the Bruhat order given in Section 2. For example, if $\bar{J}=\left\{(\emptyset ; \emptyset),\left(e_{1}-e_{2}, 2 e_{2}, e_{3}-e_{4}, 2 e_{4} ; e_{1}-e_{3}, e_{2}-e_{4}\right)\right\}$, which corresponds to the $t=\left(\begin{array}{lll}\emptyset, & 1 & 2 \\ 3 & 4\end{array}\right)$, then $\widetilde{t}=\left(\begin{array}{lll}\emptyset, & 1 & 3 \\ 2 & 4\end{array}\right)$ and so we have $\widetilde{\bar{J}}=$ $\left\{(\emptyset ; \emptyset),\left(e_{1}-e_{3}, 2 e_{3}, e_{2}-e_{4}, 2 e_{4} ; e_{1}-e_{2}, e_{3}-e_{4}\right)\right\}$ which is $W$-conjugate to the $\bar{J}$. If $w=w_{1} \in W$, then $w \bar{J}=\left\{(\emptyset ; \emptyset),\left(e_{2}-e_{1}, 2 e_{1}, e_{3}-e_{4}, 2 e_{4} ; e_{2}-e_{3}, e_{1}-e_{4}\right)\right\}$ is column standard but not row standard. Thus $\mathcal{G}_{w \bar{J}}=e-w_{1}+w_{2} w_{1}$ is the Garnir element associated with $w \bar{J}$. By Proposition 3.10 we have the Garnir relation $\mathcal{G}_{w \bar{J}} e_{w \bar{J}}=0$, so that $e_{w_{1} \bar{J}}=e_{e \bar{J}}-e_{w_{2} \bar{J}}\left(e, w_{1}, w_{2} \in D_{\Psi^{\prime}}\right)$, which has no Bruhat order relation (since $w_{1}$ appears on the left-hand side and $w_{2}$ appears on the right-hand side). But for $\widetilde{w}=w_{1} w_{2} \in W$ we have $w \bar{J}=\widetilde{w} \widetilde{J}$ and $\mathcal{G}_{w \bar{J}} e_{\widetilde{w} \widetilde{J}}=0$. Thus $e_{w_{1} w_{2} \widetilde{J}}=e_{w_{2} \widetilde{J}}-e_{e \widetilde{J}}\left(e, w_{2}, w_{1} w_{2} \in D_{\widetilde{\Psi}^{\prime}}\right)$, and we have $w_{2}<w_{1} w_{2}$ and $e<w_{1} w_{2}$. (Note that $w_{2} \widetilde{\bar{J}}=e \bar{J}$ and $e \widetilde{\bar{J}}=w_{2} \bar{J}$ are standard $\Delta$-tableaux since $e, w_{2} \in D_{\Psi} \cap D_{\Psi^{\prime}}$.) Furthermore, since $\bar{J}=w_{2} \widetilde{\bar{J}}$ for $w_{2} \in W$, then $J^{\prime}=w_{2} \widetilde{J}^{\prime}$ and so $\Psi^{\prime}=w_{2} \widetilde{\Psi}^{\prime}$. On the other hand, since $w \bar{J}$ is column standard for $w=w_{1} \in W$, then $w \in D_{\Psi^{\prime}}=D_{w_{2} \widetilde{\Psi}^{\prime}}$.

With the help of Remark 3.13, we shall now use the Garnir relations and alternacy relations to prove that any polytabloid can be written as a linear combination of standard polytabloids. We have already shown how to do this in Example 3.12 .

Theorem 3.14 If $\bar{J}$ is a very good system in $\Phi$ with notation as in (1.2), then the set $B^{\Delta, \Delta^{\prime}}=\left\{e_{w \bar{J}} \mid w \bar{J}\right.$ is a standard $\Delta-$ tableau $\}$ spans $S^{\Delta, \Delta^{\prime}}$.

Proof Let $w \bar{J}$ be any $\Delta$-tableau associated with $e_{w \bar{J}}$, where $w \in W$. Then we may assume that $e_{w \bar{J}}$ may be written as a linear combination of column standard polytabloids by Lemma 3.1. Thus, because of Remark 3.4, we may always take $w \bar{J}$ to have standard columns. Suppose that $w \bar{J}=\left\{\left(w J^{(1)} ; w J^{\prime(1)}\right),\left(w J^{(2)} ; w J^{\prime(2)}\right)\right\}$ is not row standard. This means that $w J^{(i)}$ is not row standard, where $i=1$ or 2 .

Now, take another standard $\Delta$-tableau $\widetilde{\bar{J}}$ in $\Phi$ which is $W$-conjugate to the $\bar{J}$ as in Remark 3.13. Then there exists $\widetilde{w} \in W$ such that $w \bar{J}=\widetilde{w} \widetilde{J}$ by Remark 3.13. By induction, we may assume that $e_{d \widetilde{J}}$ can be written as a linear combination of the polytabloids 
$e_{d^{\prime} \bar{J}}$ such that each $d^{\prime} \widetilde{J}^{(i)}$ is standard, where $i=1$ or 2 , when $[d \widetilde{\bar{J}}] \triangleleft[w \widetilde{\bar{J}}]$ and prove the same result for $e_{w \bar{J}}=e_{\widetilde{w} \widetilde{J}}$ by considering $w J^{(i)}=\widetilde{w} \widetilde{J}^{(i)}$, where $i=1$ or 2 .

Now suppose that $i=1$. Then there must be some negative roots in $w J^{(1)}$. For example, for the root $\alpha^{*} \in w J^{(1)}$, say $\alpha^{*} \in \Phi^{-}$. But then $\alpha^{*}=e_{a_{j}}-e_{b_{j}}$ with $a_{j}>b_{j}$ for some $j$. Let $s=\left(s_{\lambda}, s_{\mu}\right)$ be the $(\lambda, \mu)$-tableau which corresponds to the $w \bar{J}$. If we write $a_{k} \quad b_{k}$ for $e_{a_{k}}-e_{b_{k}} \in w J^{(1)}$, then we have the following situation in the part $s_{\lambda}$ of $s$ :

$\begin{array}{ccc}a_{1} & & b_{1} \\ a_{2} & & b_{2} \\ \vdots & & \vdots \\ a_{j} & > & b_{j} \\ a_{j+1} & & \vdots \\ \vdots & & b_{q} \\ a_{p} & \end{array}$

where $a_{1}<a_{2}<\ldots<a_{p}$ and $b_{1}<b_{2}<\ldots<b_{q}$ with $a_{j}>b_{j}$ for some $j$. (Here, the roots $e_{a_{1}}-e_{a_{2}}, e_{a_{2}}-e_{a_{3}}, \ldots, e_{a_{p-1}}-e_{a_{p}}, 2 e_{a_{p}}$ and $e_{b_{1}}-e_{b_{2}}, e_{b_{2}}-e_{b_{3}}, \ldots, e_{b_{q-1}}-e_{b_{q}}, 2 e_{b_{q}}$ belong to $w J^{\prime(1)}$.)

Now, take $J^{-\alpha^{*}}=\left\{\gamma \in w J^{\prime} \mid\left(\gamma,-\alpha^{*}\right) \leq 0\right\}$ then $J_{-\alpha^{*}}=\left\{-\alpha^{*}\right\} \cup J^{-\alpha^{*}}$ yields the subsystem $\Psi_{-\alpha^{*}}$ of $\Phi$ with $W\left(\Psi_{-\alpha^{*}}\right)=W\left(J_{-\alpha^{*}}\right)$ by Proposition 3.6. Furthermore, the following Dynkin diagram

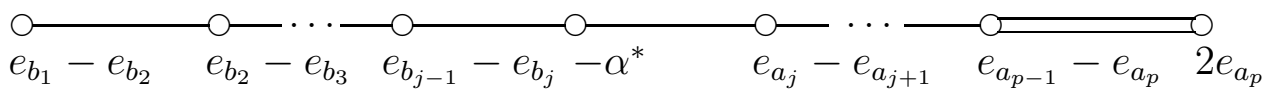

is one of the indecomposable components of $J_{-\alpha^{*}}$. Thus, if $\pi \in W\left(w J^{\prime}\right)$ then $\pm e_{a_{k}} \pm e_{b_{l}} \in$ $\pi w J^{(1)} \cap \Psi_{-\alpha^{*}}$, for some $k \in\{j, j+1, \ldots, p\}$ and $l \in\{1,2, \ldots, j\}$. Therefore, we have $\pi w \Psi \cap \Psi_{-\alpha^{*}} \neq \emptyset$ for all $\pi \in W\left(w J^{\prime}\right)$. Now, consider the corresponding Garnir element $\mathcal{G}_{w \bar{J}}=\Sigma_{\sigma}(\operatorname{sgn} \sigma) \sigma$. Then by Proposition 3.10, we have $\mathcal{G}_{w \bar{J}} e_{w \bar{J}}=0$, so that

$$
e_{w \bar{J}}=-\sum_{\sigma \neq e}(\operatorname{sgn} \sigma) e_{\sigma w \bar{J}}
$$

Since $w \bar{J}=\widetilde{w} \widetilde{\bar{J}}(w, \widetilde{w} \in W)$ then we can express this equality as

$$
e_{\widetilde{w} \widetilde{J}}=-\sum_{\sigma \neq e}(\operatorname{sgn} \sigma) e_{\sigma \widetilde{w} \widetilde{J}}
$$

which makes the tableaux $\sigma \widetilde{\widetilde{w}}$ closer to being standard than $\widetilde{\widetilde{w}}$. But the elements $\sigma \neq e$ send the tableau $\widetilde{w} \widetilde{J}$ to ones strictly smaller than $\widetilde{w} \widetilde{J}$; that is, $[\sigma \widetilde{w} \widetilde{J}] \triangleleft[\widetilde{w} \widetilde{J}]$ for all $\sigma \neq e$ by Remark 3.13. Hence, the result follows from our induction hypothesis for the part $w J^{(1)}$ of $w \bar{J}$. If the part $w J^{(2)}$ of $w \bar{J}$ is also not row standard, then there must again be some negative roots in $w J^{(2)}$. For example, suppose that $\beta^{*} \in w J^{(2)}$ with $\beta^{*} \in \Phi^{-}$. 
Then $\beta^{*}$ is either a negative short root or a negative long root. If $\beta^{*}$ is a negative short root, then the result can be deduced when we repeat the above process by considering the part $w J^{(2)}$ of $w \bar{J}$. But if $\beta^{*}$ is a negative long root then $\beta^{*}=-2 e_{i}$ for some $i$. Thus, Remark 3.11 yields $e_{w \bar{J}}=e_{w_{-\beta^{*}} w \bar{J}}$. Since we have $w \bar{J}=\widetilde{w} \widetilde{J}$ then we can express this equality as $e_{\widetilde{w} \widetilde{J}}=e_{w_{-\beta^{*}} \widetilde{w} \widetilde{J}}$ (clearly, $w_{-\beta^{*}} \widetilde{w} \bar{J}$ is closer to being standard than $\widetilde{w} \widetilde{J}$ ), where $\left[w_{-\beta^{*}} \widetilde{w} \widetilde{J}\right] \triangleleft[\widetilde{w} \widetilde{J}]$ by Remark 3.13. Therefore, the result follows from our induction hypothesis for the part $w J^{(2)}$ of $w \bar{J}$. Hence, we have the required result.

Corollary 3.15 If $\bar{J}$ is a very good system in $\Phi$ with notation as in (1.2), then the set $B^{\Delta, \Delta^{\prime}}$ is a $K$-basis for $S^{\Delta, \Delta^{\prime}}$, and $\bar{J}$ is therefore a perfect system in $\Phi$.

Proof The result follows from Proposition 2.3 and Theorem 3.14.

This paper may be taken to be the proposal of a recipe for Garnir elements in terms of the root systems; that is, the construction of the Garnir elements proposed here may be potentially applied to other Weyl groups. (Of course, the Garnir elements constructed in this paper are valid for the Weyl groups of type $A_{n}$ if $\bar{J}$ is a very good system in $\Phi=A_{n}$ obtained from the standard $\lambda$-tableau as in (1.2).) In the following example, we show how a Garnir relation can be constructed for the Weyl group of type $D_{4}$. In a future publication, the method presented in this work for obtaining Garnir elements will be extended to the Weyl group of any type.

Example 3.16 Referring to Example 3.24 of [10], let $\Phi=D_{4}$ with simple system $\pi=$ $\left\{\alpha_{i}=e_{i}-e_{i+1}(i=1,2,3), \alpha_{4}=e_{3}+e_{4}\right\}$ and corresponding Weyl group $W=W\left(D_{4}\right)$. Let $w_{\alpha_{i}}$ be denoted by $w_{i}, i=1,2,3,4$. Let $\Psi=A_{3}$ be a subsystem of $D_{4}$ with simple system $J=\left\{e_{1}-e_{2}, e_{2}-e_{3}, e_{3}-e_{4}\right\}$ and let $\Psi^{\prime}=2 A_{1}$ be a subsystem of $D_{4}$ with simple system $J^{\prime}=\left\{e_{1}+e_{4}, e_{2}+e_{3}\right\}$. Then $\bar{J}=\left\{e_{1}-e_{2}, e_{2}-e_{3}, e_{3}-e_{4} ; e_{1}+e_{4}, e_{2}+e_{3}\right\}$ is a good system in $\Phi$ and the corresponding Specht module $S^{\Delta, \Delta^{\prime}}$ has dimension 3. The set of standard $\Delta$-polytabloids is $B^{\Delta, \Delta^{\prime}}=\left\{e_{\bar{J}}, e_{w_{4} \bar{J}}, e_{w_{2} w_{4} \bar{J}}\right\}$. By definition of a very good system, it follows that $\bar{J}$ is a very good system in $\Phi$, and so the set $B^{\Delta, \Delta^{\prime}}$ is linearly independent over $K$ by Proposition 2.3.

Now, let $w \bar{J}$ be any $\Delta$-tableau associated with $e_{w \bar{J}}$, where $w \in W$. Then we may always assume that $w \bar{J}$ is column standard (since alternacy relation is valid for every Weyl group). If $w=w_{1} w_{4} \in W$, then $w \bar{J}=\left\{e_{2}-e_{1}, e_{1}+e_{4}, e_{3}-e_{4} ; e_{2}-e_{3}, e_{1}-e_{4}\right\}$ is a $\Delta$-tableau. Since $\alpha^{*}=e_{2}-e_{1}$ is a negative root in $w \bar{J}, w \bar{J}$ is not row standard. Now, take $J^{-\alpha^{*}}=\left\{e_{2}-e_{3}\right\}$ then $J_{-\alpha^{*}}=\left\{-\alpha^{*}\right\} \cup J^{-\alpha^{*}}=\left\{e_{1}-e_{2}, e_{2}-e_{3}\right\}$ yields the subsystem $\Psi_{-\alpha^{*}}=A_{2}$ of $\Phi$ with $W\left(J_{-\alpha^{*}}\right)=\left\langle w_{1}, w_{2}\right\rangle$ by Proposition 3.6. Since $W\left(w J^{\prime}\right)=\left\langle w_{2}\right\rangle \times\left\langle w_{1} w_{2} w_{3} w_{2} w_{1}\right\rangle$, we have $S=W\left(J_{-\alpha^{*}}\right) \cap W\left(w J^{\prime}\right)=\left\langle w_{2}\right\rangle$.

Let $e, w_{1}, w_{2} w_{1}$ be coset representatives for $S$ in $W\left(J_{-\alpha^{*}}\right)$. (Note that at this point $e w \bar{J}, w_{1} w \bar{J}, w_{2} w_{1} w \bar{J}$ are all the column standard tableaux.) Then $\mathcal{G}_{w \bar{J}}=e-w_{1}+w_{2} w_{1}$ is the Garnir element associated with $w \bar{J}$. Moreover, we have $\pi w \Psi \cap \Psi_{-\alpha^{*}} \neq \emptyset$ for all $\pi \in W\left(w J^{\prime}\right)$ and so $\mathcal{G}_{w \bar{J}} e_{w \bar{J}}=0$, so that $e_{w_{1} w_{4} \bar{J}}=e_{w_{4} \bar{J}}-e_{w_{2} w_{4} \bar{J}}\left(w_{1} w_{4}, w_{4}, w_{2} w_{4} \in D_{\Psi^{\prime}}\right)$. Now, take another standard $\Delta$-tableau $\widetilde{\bar{J}}=w_{2} w_{4} \bar{J}$. Clearly, $\widetilde{\bar{J}}$ is $W$-conjugate to the $\bar{J}$. 
Then for $\widetilde{w}=w_{1} w_{2} \in W$ we have $w \bar{J}=\widetilde{w} \widetilde{J}$ and so $\mathcal{G}_{w \bar{J}} e_{\widetilde{w} \widetilde{J}}=0$. Thus $e_{w_{1} w_{2} \widetilde{J}}=e_{w_{2}} \widetilde{J}-e_{e \widetilde{J}}$ $\left(e, w_{2}, w_{1} w_{2} \in D_{\widetilde{\Psi}^{\prime}}\right)$, where $w_{2}<w_{1} w_{2}$ and $e<w_{1} w_{2}$. (Here, note that $w_{2} \widetilde{\bar{J}}=w_{4} \bar{J}$ and $e \widetilde{\bar{J}}=w_{2} w_{4} \bar{J}$ are standard $\Delta$-tableaux.)

By a similar calculation to the above, it can be shown that any polytabloid can be written as a linear combination of standard polytabloids. Hence, the set $B^{\Delta, \Delta^{\prime}}=$ $\left\{e_{\bar{J}}, e_{w_{4} \bar{J}}, e_{w_{2} w_{4} \bar{J}}\right\}$ is a $K$-basis for $S^{\Delta, \Delta^{\prime}}$.

We conclude this paper with a difficult question. Let $(\lambda, \mu)$ be a pair of partitions of $n$ such that $\lambda$ is a partition of $|\lambda|$ and $\mu$ is a partition of $|\mu|$, and $|\lambda|+|\mu|=n$. Many results about representations of the hyperoctahedral groups can be approached in a purely combinatorial manner. The crucial link between these two viewpoints is the fact that the dimension of the Specht module $S^{\lambda, \mu}$ is the number of standard $(\lambda, \mu)$-tableaux (see Theorem 2.18 in [14]). If $\bar{J}$ is a very good system in $\Phi$ with notation as in (1.2), then the Specht modules $S^{\Delta, \Delta^{\prime}}, S^{\lambda, \mu}$ are isomorphic. Now, let $h_{\lambda}, h_{\mu}$ be the product of all the hooklengths in the diagrams $[\lambda]$, $[\mu]$, respectively, and let $h_{\lambda, \mu}=h_{\lambda} h_{\mu}$. To obtain a standard $(\lambda, \mu)$-tableau, choose $|\lambda|$ elements from $\{1,2, \ldots, n\}$, construct a standard $\lambda$-tableau from them, and construct a standard $\mu$-tableau from the remainder. Thus

$$
\operatorname{dim}_{K} S^{\Delta, \Delta^{\prime}}=\frac{n !}{|\lambda| !|\mu| !} \frac{|\lambda| !}{h_{\lambda}} \frac{|\mu| !}{h_{\mu}}=\frac{n !}{h_{\lambda, \mu}} \text { (hook formula). }
$$

Question: How can we describe the above hook formula in terms of root systems?

\section{Acknowledgement}

I would like to express my appreciation to Professor A. O. Morris at the University of Wales, Aberystwyth for guiding me into this area of research.

\section{References}

[1] E. Al-Aamily, A. O. Morris and M. H. Peel, The representations of the Weyl groups of type $B_{n}$, J. Algebra 68 (1981), 298-305.

[2] H. Can, Representations of the generalized symmetric groups, Contrib. Alg. Geom. 37(2) (1996), 289-307.

[3] H. Can, Some combinatorial results for complex reflection groups, Europ. J. Combinatorics 19 (1998), 901-909.

[4] H. Can, On the perfect systems of the Specht modules of the Weyl groups of type $C_{n}$, Indian J. pure appl. Math. 29(3) (1998), 253-269.

[5] R. W. Carter, Simple Groups of Lie Type, Wiley, London, New York, Sydney, Toronto, 1989.

[6] A. M. Cohen, Finite complex reflection groups, Ann. Sci. Ec. Norm. Sup. 4(9) (1976), $379-436$. 
[7] E. B. Dynkin, Semisimple subalgebras of semisimple Lie algebras, Amer. Math. Soc. Transl. 6(2) (1957), 111-244.

[8] S. Halicioglu, A basis for Specht modules for Weyl groups, Turkish J. of Math. 18 (1994), 311-326.

[9] S. Halicioglu, The Garnir relations for Weyl groups, Math. Japonica 40(2) (1994), 339-342.

[10] S. Halicioglu and A. O. Morris, Specht modules for Weyl groups, Contrib. Alg. Geom. 34(2) (1993), 257-276.

[11] J. E. Humphreys, Reflection Groups and Coxeter Groups, Cambridge Studies in Advanced Math. 29, Cambridge University Press, Cambridge, New York, 1990.

[12] A. J. Idowu and A. O. Morris, Some combinatorial results for Weyl groups, Math. Proc. Camb. Phil. Soc. 101 (1987), 405-420.

[13] G. D. James, The Representation Theory of the Symmetric Groups, Lecture Notes in Math., Vol. 682, Springer-Verlag, New York, 1978.

[14] A. O. Morris, Representations of Weyl groups over an arbitrary field, Asterisque 87-88 (1981), 267-287.

[15] M. H. Peel, Specht modules and symmetric groups, J. Algebra 36 (1975), 88-97.

[16] B. E. Sagan, The Symmetric Group-Representations, Combinatorial Algorithms and Symmetric Functions, Wadsworth \& Brooks/Cole, Pacific Grove, California, 1991. 